months as a senior house officer and the experiences described mirrored mine exactly.

The most enjoyable aspects were being able to work without the interruption of a bleep, enabling one to concentrate wholeheartedly, and becoming part of a new team whose approach was so different from any in a hospital setting. What struck me was that although each person worked autonomously he or she also contributed significantly to the whole team, either in the weekly referral meeting or during daily work.

My experiences have made me more aware of patients' family and social circumstances which can easily be forgotten in a busy acute unit and I would strongly recommend that all trainees spend at least six months working in a child guidance clinic.

HELEN TUCKER, Torbay Psychiatric Rotation Scheme, Edth Morgan Unit, Torbay Hospital, Torquay TQ2 7AA

Sir: I was interested to read Jan Hermsen's description of work in a child guidance clinic. (Psychiatric Bulletin, 1993, 17, 626). I am currently working as a registrar in a child and adolescent unit and would agree that an experience in this area is essential for all trainees. The shift to a totally different patient population with their own peculiar set of disorders requires a major rethink of approach, method and technique which can be revealing and, at times, painful.

In this area of psychiatry it is acknowledged that the patient comes as part of a package, i.e. the family. You cannot treat the one without the other. In the clinic in which I work family therapy is a major part of the treatment offered. It has opened my eyes to the importance of an approach neglected or ignored in other areas of psychiatry.

Child and adolescent psychiatry involves the treatment of largely unwilling and involuntary patients who are frequently unable to articulate their problems. The predominantly psychotherapeutic basis of this treatment can be unappealing or alarming to the practitioner. However, trainees can gain vital skills which may not be possible in other specialities.

S. C. CARvill, 14 Woodloes Road, Shirley, Solihull, West Midlands B9O 2RP

\section{Psychiatric emergencies}

Sir: I read with interest Kohen's paper on psychiatric emergencies in people with a mental handicap (Psychiatric Bulletin, 1993, 17, 587-589). We have just completed a survey of all psychiatric emergencies in the Borders region over one year (June 1992 to May 1993). In contrast to Kohen's survey of emergencies seen by a consultant during the daytime in an urban area, most of our cases were seen out of hours by junior doctors and came from a dispersed rural population of 104,000 .

During the year there were 13 emergencies with a diagnosis of learning difficulty. This was $2.7 \%$ of all psychiatric emergencies. The mean age was 36 with a range of 20-69 years. Eleven were male. Only one of the 13 was seen by a consultant, the others being seen by junior doctors, usually with a co-therapist. Eleven were seen out of hours and all were seen within four hours of referral. The commonest source of referral was from the GP (six cases), and the commonest site of assessment was at home (six cases). Referral was precipitated by self-harm in only one case. Most of the patients had never been married (ten cases), but only one still lived with his parents. The others lived alone (six cases) or with other people (six cases). Just over half were employed full or part-time (seven cases).

Nine patients had another diagnosis apart from learning difficulties. There were five cases of neurotic and stress-related disorders, three of affective psychosis and one of personality disorder. Unlike Kohen's study, none were referred for epilepsy. All the referrals had a history of previous admissions and ten were active cases. The consultant was contacted in only two cases, both times to discuss the decision to admit. Six patients were admitted, the reasons given including risk to self, risk to others, social factors, for assessment and for treatment. Eleven cases were thought to be appropriate emergency referrals.

Only two cases were referred on Saturday and there was no evidence of a 'Saturday afternoon syndrome' as described by Spenser (Psychiatric Bulletin, 1993, 17, 565). This may reflect better local support from the social work department or other differences in local practice. We would not dispute her conclusion that it is important to have provision of emergency short-term beds for assessment and treatment of people with learning difficulties. We had only 13 in-patient beds at the time of this study and would also support Kohen's conclusion that anxiety about emergencies should not deter the implementation of community care. Most of the referrals were appropriate and the actual demand was small and, to a large extent, met by existing out-of-hours arrangements.

JoHN TAYLOR, Dingleton Hospital, Melrose, Roxburghshire TD6 9HN

\section{Defeat Depression Campaign}

Sir: The thesis of the Defeat Depression Campaign is that patients are reluctant to take 
psychological symptoms to their general practitioners and that consequently, opportunities for prevention and early diagnosis are being missed. This is demonstrated in the surveys described by Vize \& Priest. (Psychiatric Bulletin, 1993, 17, 573-574). As a profession we have made a working hypothesis that this represents a transference phenomenon related to patients' beliefs and attitudes about mental illness, and have predicted that health education will help to overcome their barrier to early intervention. We have also advocated screening in primary care settings, which as doctor-initiated consultations may depersonalise sensitive issues and remove the individual threat which inhibits patients from seeking help. Psychiatrists, however, continue to debate the diagnostic value of rating scales (Snaith, 1993), and I fear that many GPs remain unconvinced about the value of using such instruments during health checks.

An example of this is the assessment of the mental state of over-75-year-olds during annual health checks which were introduced as part of the GP contract in 1990 . There is wide variation in the organisation and content of these checks, but where mental state is assessed by rating instruments, up to $30 \%$ of patients have significant symptoms (Iliffe et al, 1991).

I have recently surveyed the content of over-75 health checks in 30 randomly selected GP practices in Norfolk. Twenty-three $(77 \%)$ responded, of which only one $(4.3 \%)$ routinely used a depression rating scale and only three (13.0\%) a scale assessing cognitive function. Thirteen practices (56.5\%) based their assessments on a general impression without any specific questioning; $70 \%$ of health checks were performed by nurses and health visitors.

Opportunities for prevention and early diagnosis are being missed. While in some cases there may be resource or training deficits, it seems likely that the case for screening has not been proven sufficiently to establish the priority for service provision. Shepherd (1991) highlighted the need for research and co-operation between GPs and psychiatrists. The paradigm and numbers needed to clarify issues of interpreting rating scales and their application as screening instruments in primary care lies exactly there, in general practice, where the story of depression usually begins and ends.

IlfFFe, S., Haines, A., Gallivan, S., Booroff, A. et al (1991) Assessment of elderly people in general practice. 1. Social circumstances and mental state. British Journal of General Practice, 41, 9-12.

SHEPHERD, M. (1991) Primary care psychiatry: the case for action. British Journal of General Practice, 41, 252-255.

SNATT, P. (1993) What do depression rating scales measure? British Journal of Psychiatry, 163, 293-298.

Michael, J. Noble, Little Plumstead Centre, Norwich NR13 5EW

\section{Italian mental health law}

Sir: Dr Rollin's article, ‘Community Care: Italy's "U" Turn' (Psychiatric Bulletin, 17, 494-495, 1993) reminded me of my recent study tour of the Fiorenzuola and Lombardy regions in Northern Italy. I realise that this limits my perspective and I will refrain from generalising too much. Jones (1991) argues that it is impossible to generalise about 'The Italian Experience'. Services in one district can be quite different from those in the next.

I heartily agree with Dr Rollin. The hospitality I experienced was excellent.

The principal goals of Law 180/78 included creating a decentralised community service of preventing mental illness and treating and rehabilitating mental patients and promoting comprehensive treatment, particularly through services outside a hospital framework (Cassana et al, 1985).

Law 180/78 actually succeeded in introducing significant change in the provision of psychiatric care. The emphasis has shifted from protection of the public towards better meeting of patients' needs through community care. New admissions to the 'old style' psychiatric hospitals ceased immediately. The law required re-admissions to cease without two years. Nobody was discharged 'willy-nilly' into the community. Psychiatrically ill patients are still admitted, mostly to 15 bed diagnosis and care units in general hospitals. The total number of admissions to these units was 54,104 in 1984; 79\% were voluntary admissions (Crepet, 1990).

But I do agree with Dr Rollin that there is a lesson for Mrs Bottomley. Professor Franco Rotelli has agreed that Law 180 falled because the political will never existed to implement it (Endean, 1993). But of even greater relevance to our own NHS, Signora Basaglia, (Franco Basaglia's widow) has consistently blamed the failure of law 180 on lack of resources.

I sincerely hope that the lessons for the Italian Experience becomes mandatory reading for those charged with implementing community care in the UK.

Cassano, G.B., Mauri, M. \& Petracca, A. (1985) The current status of psychiatric care in Italy. International Journal of Mental Health. 14, 174-183.

CREPET, P. (1990) A transition period in psychiatric care in Italy ten years after the reform. British Journal of Psychiatry. 156, 27-36.

ENDEAN, C. (1993) Italy retreats from community care for mentally ill. British Medical Journal, 306, 605.

JONES. K. (1991) The Italian Mental Health Law. British Journal of Psychiatry, 159, 556-557.

O. JUNAID, Queen's Medical Centre, University Hospital, Nottingham NG7 2UH 